\title{
Long Noncoding RNAs CARMN, LUCAT1, SMILR, and MALAT1 in Thoracic Aortic Aneurysm: Validation of Biomarkers in Clinical Samples
}

\author{
Vaiva Patamsytė ${ }^{D},{ }^{1}$ Giedrius Žukovas, ${ }^{2}$ Dovydas Gečys, ${ }^{1}$ Diana Žaliaduonyte், ${ }^{3}$ \\ Povilas Jakuška, ${ }^{2}$ Rimantas Benetis, ${ }^{1,2}$ and Vaiva Lesauskaitė $\mathbb{D}^{1}$ \\ ${ }^{1}$ Institute of Cardiology, Lithuanian University of Health Sciences, Sukilèliu pr. 15, Kaunas LT-50103, Lithuania \\ ${ }^{2}$ Department of Cardiac, Thoracic and Vascular Surgery, Lithuanian University of Health Sciences, Eiveniu g. 2, \\ Kaunas LT-50161, Lithuania \\ ${ }^{3}$ Department of Cardiology, Lithuanian University of Health Sciences, Eiveniu g. 2, Kaunas LT-50161, Lithuania
}

Correspondence should be addressed to Vaiva Patamsytė; vaiva.patamsyte@lsmuni.lt

Received 22 February 2020; Revised 26 May 2020; Accepted 6 June 2020; Published 17 June 2020

Academic Editor: Zhongjie Shi

\begin{abstract}
Copyright (c) 2020 Vaiva Patamsyte et al. This is an open access article distributed under the Creative Commons Attribution License, which permits unrestricted use, distribution, and reproduction in any medium, provided the original work is properly cited.
\end{abstract}

\begin{abstract}
Background and Objectives. Thoracic aortic aneurysm (TAA) is a silent disease characterised by aortic wall expansion and vascular smooth muscle cell (VSMC) dedifferentiation from contractile to synthetic phenotype. Long noncoding RNAs (lncRNAs) involved in VSMC phenotypic regulation could be considered as potential diagnostic indicators and therapeutic targets of TAA. In vitro studies show that lncRNAs CARMN, LUCAT1, MALAT1, and SMILR are associated with the VSMC phenotypic state. Our aim was to test if these lncRNAs are dysregulated during TAA formation in clinical patient samples. Materials and Methods. Relative expression of lncRNAs CARMN, LUCAT1, SMILR, and MALAT1 was tested in clinical aortic tissue and blood plasma samples from TAA and non-TAA patients using the qRT-PCR method. The Mann-Whitney $U$ test was used to compare $\Delta$ Ct values between the study groups. ROC curve analysis was performed to evaluate the diagnostic value of plasma lncRNAs. Results. We found significantly reduced CARMN $(p=0.033)$ and LUCAT1 $(p=0.009)$ expression in aortic tissue samples from TAA patients. Relative expression of MALAT1 $(p=0.117)$ and SMILR $(p=0.610)$ did not differ in aortic tissue between the TAA and non-TAA groups. Expression of both LUCAT1 and SMILR was significantly decreased in TAA patients' blood plasma compared to controls $(p=0.018$ and $p=0.032$, respectively). However, only LUCAT1 showed the ability to discriminate aneurysmal disease in patients' blood plasma ( $\mathrm{AUC}=0.654,95 \% \mathrm{CI}=0.534-0.775, p=0.018$ ). Conclusions. We have shown that the expression of lncRNAs CARMN and LUCAT1 is reduced in dilated aortic tissue and that the LUCAT1 and SMILR expression is lower in the blood plasma of TAA patients. Decreased LUCAT1 expression in TAA patients' blood plasma may have diagnostic potential in discriminating patients with TAA.
\end{abstract}

\section{Introduction}

Thoracic aortic aneurysm (TAA) is mainly an asymptomatic disease with an increasing incidence rate [1]. Biomarker research has long been a focus for the development of an effective clinical screening for TAA [2]. Sequencing technologies lead to identification of various noncoding RNAs which are considered key players in the regulation of biological processes. A class of small noncoding RNAs called microRNAs
(miRNAs) is extensively studied in the development of cardiovascular diseases (CVDs) as potential biomarkers and therapeutic targets [3]. Intriguing information begins to emerge regarding the role of long noncoding RNAs (lncRNAs), but these studies are still limited, and specific contributions of lncRNAs to the disease development and progression remain unexplored [4]. IncRNAs are transcripts larger than $200 \mathrm{nt}$ and exert different cellular function depending on their localization in the nucleus or cytoplasm 
$[5,6]$. TAA shows common histological features such as the vascular smooth muscle cell (VSMC) phenotype switch from contractile to synthetic and extracellular matrix remodelling [7]. Several mechanisms are revealed by which lncRNAs control VSMC migration [8], proliferation [9], and angiotensin II (Ang II) signalling system [10]. Due to their regulatory capabilities, lncRNAs might play an important role in aortic aneurysmal disease [11].

Circulating lncRNAs show a diagnostic potential in coronary artery disease $[12,13]$, acute myocardial infarction [14], diabetic cardiomyopathy [15], and chronic heart failure [16]. A study on the rat model has reported increased expression of specific lncRNAs in both plasma and cardiac samples during acute myocardial infarction [14]. Recent in vitro studies identify lncRNAs, associated with the VSMC phenotypic state, namely, metastasis-associated lung adenocarcinoma transcript 1 (MALAT1) [17], smooth muscleinduced lncRNA (SMILR), cardiac mesoderm enhancerassociated lncRNA (CARMN), and lung cancer-associated transcript 1 (LUCAT1) [18]. Using clinical samples, we test if these lncRNAs are differentially expressed in the aortic wall and blood plasma of TAA patients and individuals without aortic dilatation. This information will help to assess the potential of MALAT1, SMILR, CARMN, and LUCAT1 to be used as biomarkers for the formation of TAA.

\section{Materials and Methods}

2.1. Study Subjects. Study subjects were recruited at the Hospital of Lithuanian University of Health Sciences, Department of Cardiology and Department of Cardiac, Thoracic and Vascular Surgery during the period of 2016-2019. Aortic tissue samples $(N=24)$ and blood plasma samples $(N=40)$ were obtained from sporadic TAA patients, who were diagnosed with aortic aneurysm in accordance with 2014 ESC guidelines on the diagnosis and treatment of aortic disease [19]. Aortic tissue samples of the non-TAA group $(N=42)$ consisted of heart donors $(N=5)$ without a history of cardiovascular pathology and patients $(N=37)$ who had an isolated coronary artery bypass procedure (CABG) without aortic dilation. Non-TAA blood plasma samples $(N=53)$ were collected from study subjects, who did not have a diagnosis of TAA. All study subjects except heart donors had twodimensional thoracic echocardiography. Our study excluded cases of severe atherosclerosis of the ascending aorta $(N=3)$, aortitis $(N=4)$, and genetic syndromes $(N=2$, both Marfan). The study was approved by the Kaunas Regional Biomedical Research Ethics Committee (Nr. P2-BE-2-12/2012) in accordance with the Declaration of Helsinki.

2.2. Sample Collection. Tissue samples from TAA patients were collected from convexity of the ascending aorta. Segments of $5 \times 5 \mathrm{~mm}$ in size were cut out from the outer curvature. Aortic specimens from CABG patients were taken from the ascending aorta at the site of proximal bypass anastomosis (punch biopsies). Ascending aorta samples from donors were taken during the heart transplantation procedure. All tissue samples were immediately put into RNAlater ${ }^{\mathrm{TM}}$ (Thermo Fisher Scientific, Lithuania) stabilization solution, kept at $+4^{\circ} \mathrm{C}$ overnight, and later stored at $-80^{\circ} \mathrm{C}$. Whole blood samples from TAA and non-TAA study subjects were collected into $3 \mathrm{ml}$ Venosafe $^{\mathrm{TM}}$ vacutainer tubes with K2EDTA (Terumo Europe, Belgium) before heparinization. Blood plasma was prepared within an hour of phlebotomy procedure by centrifugation at $1,900 \mathrm{~g}$, for $10 \mathrm{~min}$. Plasma samples were stored at $-80^{\circ} \mathrm{C}$.

2.3. RNA Extraction and Quantification. Aortic tissue samples were frozen with liquid nitrogen and manually ground for total RNA extraction using a mirVana ${ }^{\mathrm{TM}}$ miRNA Isolation Kit (Thermo Fisher Scientific, Lithuania) and Acid Phenol: $\mathrm{CHCl} 3$ premix (Ambion, USA) according to the manufacturer's protocol. RNase Inhibitor (Applied Biosystems, USA) was added to a lysis buffer to prevent RNA degradation. RNA concentration and integrity were assessed using a NanoDrop 2000 Spectrophotometer (Thermo Scientific) and RNA 6000 Nano Kit (Agilent Technologies, USA) on a 2100 Bioanalyzer System (Agilent). RNA samples were treated with a DNase TURBO DNA-free ${ }^{\mathrm{TM}}$ Kit (Thermo Fisher Scientific, Lithuania) to eliminate any DNA contamination. A miRNeasy Serum/Plasma Kit (QIAGEN, Germany) was used for total RNA extraction from plasma samples, according to the manufacturer's protocol. Reverse transcription for lncRNA expression analysis was done with High Capacity RNA-to-cDNA Kit (Applied Biosystems, USA).

Gene expression experiments were done according to the manufacturer's protocol on an ABI 7900HT Fast Real Time PCR System (Applied Biosystems) using TaqMan ${ }^{\circledR}$ Fast Advanced Master Mix (Applied Biosystems, USA) and TaqMan Assays. Expression of lncRNAs was evaluated using TaqMan ${ }^{\circledR}$ Gene Expression Assays for MALAT1 (assay ID Hs00273907_s1), CARMN (assay ID Hs04402463_ m1), LUCAT1 (assay ID Hs00884761_s1), and a custommade SMILR assay with forward primer sequence $5^{\prime}$-GGAT ATGAATTGTAATGGCCAGAGCAT-3', reverse primer sequence $5^{\prime}$-GAATTCAGTCTTGGTTCCCTAAAATGG-3', and a reporter dye FAM with sequence CTGTGAGATGA AAACTC. Expression level of glyceraldehyde 3-phosphate dehydrogenase (GAPDH) [15] (assay ID Hs99999905_m1), was used as an endogenous control for lncRNA data normalisation. All $\Delta \mathrm{Ct}$ values were calculated using ExpressionSuite v1.2 Software (Thermo Fisher Scientific) and log-transformed.

2.4. Statistical Analysis. Data normality was checked using a Kolmogorov-Smirnov test. A nonparametric Mann-Whitney $U$ test was used to compare log-transformed $\Delta \mathrm{Ct}$ values of lncRNAs between the study groups. Differences in clinical characteristics were calculated using Mann-Whitney $U$ test and chi-squared test. Receiver operating characteristic (ROC) curve analysis was performed to evaluate the diagnostic value of plasma lncRNAs in TAA and non-TAA patients. GraphPad Prism 8 software (San Diego, CA, USA) was used to construct plots and perform calculations.

\section{Results}

Clinical characteristics of patients in all study groups are summarized in Table 1 . We did not find an association 
TABLE 1: Clinical characteristics of patients in all study groups.

\begin{tabular}{|c|c|c|c|c|c|c|}
\hline \multirow{2}{*}{ Characteristic } & \multicolumn{3}{|c|}{ Tissue } & \multicolumn{3}{|c|}{ Plasma } \\
\hline & $\operatorname{TAA}(N=24)$ & Non-TAA $(N=42)$ & $p$ value & TAA $(N=40)$ & Non-TAA $(N=53)$ & $p$ value \\
\hline Age, yrs median (range) & $60(43-83)$ & $70(41-86)$ & 0.376 & $65(39-87)$ & $70(41-86)$ & 0.039 \\
\hline Males, $n(\%)$ & $21(88 \%)$ & $33(79 \%)$ & 0.365 & $31(78 \%)$ & $40(75 \%)$ & 0.819 \\
\hline Bicuspid aortic valve, $n(\%)$ & $19(79 \%)$ & $1(2 \%)$ & $<0.001$ & $18(45 \%)$ & $1(2 \%)$ & $<0.001$ \\
\hline Hypertension, $n(\%)$ & $20(83 \%)$ & $36(86 \%)$ & 0.067 & $34(85 \%)$ & $49(92 \%)$ & 0.251 \\
\hline Aortic valve insufficiency, $n(\%)$ & $18(75 \%)$ & $12(29 \%)$ & $<0.001$ & $31(78 \%)$ & $17(32 \%)$ & $<0.001$ \\
\hline Aortic valve stenosis, $n(\%)$ & $10(42 \%)$ & $2(5 \%)$ & $<0.001$ & $13(33 \%)$ & $2(4 \%)$ & $<0.001$ \\
\hline Ascending aortic diameter, mm median (range) & $52(45-65)$ & $35(28-38)^{*}$ & $<0.001$ & $49(41-65)$ & $35(26-39)$ & $<0.001$ \\
\hline Aortic annulus, mm median (range) & $26(23-32)$ & $24(20-28)^{*}$ & $<0.001$ & $27(22-34)$ & $23(19-28)$ & $<0.001$ \\
\hline Aortic sinus, mm median (range) & $48(38-68)$ & $37(26-40)^{*}$ & $<0.001$ & $46(32-74)$ & $37(26-42)$ & $<0.001$ \\
\hline
\end{tabular}

${ }^{*}$ Data missing from five donors.

between IncRNA expression profiles and presence of aortic valve insufficiency and hypertension $(p>0.05)$. Only LUCAT1 expression in the aortic wall was significantly reduced in the study subjects with a bicuspid aortic valve (BAV) compared to tricuspid aortic valve (TAV) patients $(p=0.004)$. Relative expression profiles of CARMN, LUCAT1, MALAT1, and SMILR in aortic wall specimens of non-TAA and TAA patients are shown in Figure 1. Both CARMN $(p=0.033)$ and LUCAT1 $(p=0.009)$ were significantly reduced in TAA compared to non-TAA aortic tissue samples. There was no significant difference in MALAT1 $(p=0.117)$ or SMILR $(p=0.610)$ expression between TAA and non-TAA aortic tissue samples.

Expression of LUCAT1 was also significantly reduced $(p=0.018)$ in the blood plasma of TAA patients compared to the non-TAA group (Figure 2(a)). We have also observed reduced SMILR expression $(p=0.032)$ in TAA patients' blood plasma compared to that of the non-TAA group. MALAT1 expression in blood plasma did not differ between the two study groups $(p=0.087)$. Unfortunately, CARMN expression in blood plasma could not be detected using the qRT-PCR method.

A ROC curve analysis for identification of TAA was performed on LUCAT1, MALAT1, and SMILR relative expression levels in blood plasma. An area under curve $($ AUC) $>0.65$ indicates a potential diagnostic value of a certain indicator for the disease [20]. Only LUCAT1 (AUC $=0.654,95 \% \mathrm{CI}=$ $0.534-0.775, p=0.018$ ) represented an ability to discriminate aneurysmal disease in patients' blood plasma (Figure 2(b)).

\section{Discussion}

The thoracic aortic aneurysm has a clinically silent course until development of complications such as aortic dissections. Thus, identification of biomarkers for early diagnostics and clinical course prediction is of great importance and is comparable to the Holy Grail search [2]. Development of thoracic aorta aneurysm is a complex biological process involving smooth muscle cell phenotypic transition. lncRNAs CARMN, LUCAT1, MALAT1, and SMILR are associated with the VSMC phenotypic state in vitro. Our aim was to test if these $\operatorname{lncRNAs}$ are dysregulated during TAA formation in clinical patient samples. Thus, we compared expression of these lncRNAs in clinical samples from TAA and non-TAA patients.

CARMN function was first identified in cardiomyocytes as a crucial regulator of cardiac cell differentiation [21]. Ounzain and colleagues demonstrated that CARMN is a super enhancer-associated $\operatorname{lncRNA}$, highly expressed in adult mouse and human heart to maintain cardiac homeostasis and remodeling [21]. CARMN expression is increased in differentiated VSMCs [18], supporting its important role in maintaining a contractile phenotype. We have identified a significant reduction of CARMN in aortic wall samples of TAA patients compared to non-TAA study subjects. Indeed, a high-throughput sequencing analysis has previously identified a significant reduction of CARMN in the aortic wall of thoracic aortic dissection (TAD) patients as well [22]. These results suggest that CARMN plays an important role in the loss of the contractile phenotype of VSMCs and development of TAA. In our study, expression of CARMN could not be detected in patients' blood plasma using the qRT-PCR method. However, increased CARMN expression is observed in peripheral blood nuclear cells (PBNC) of hypertensive patients [23]. Further studies will be needed to test if CARMN expression in patients' PBNCs could be associated with TAA formation.

LUCAT1 was first identified in tumorigenesis as a predictor of poor prognosis in human non-small lung cancer [24]. It is described as a regulator and biomarker for cell cycle progression, proliferation, and metastasis in various cancers [25-28]. A recent article found that downregulation of LUCAT1 inhibits proliferation and promotes apoptosis of cardiomyocytes [29]. LUCAT1 also plays a part in the regulation of apoptosis in smooth muscle cells [30]. Authors show that LUCAT1 depletion is able to increase proliferation and suppress smooth muscle cell apoptosis. In our study, lower expression levels of LUCAT1 in aortic tissue samples from TAA patients could also indicate a regulatory role in VSMC dedifferentiation processes. A significant association between the type of aortic valve and LUCAT1 expression in aortic tissue suggests that it could be a significant player in BAV- 

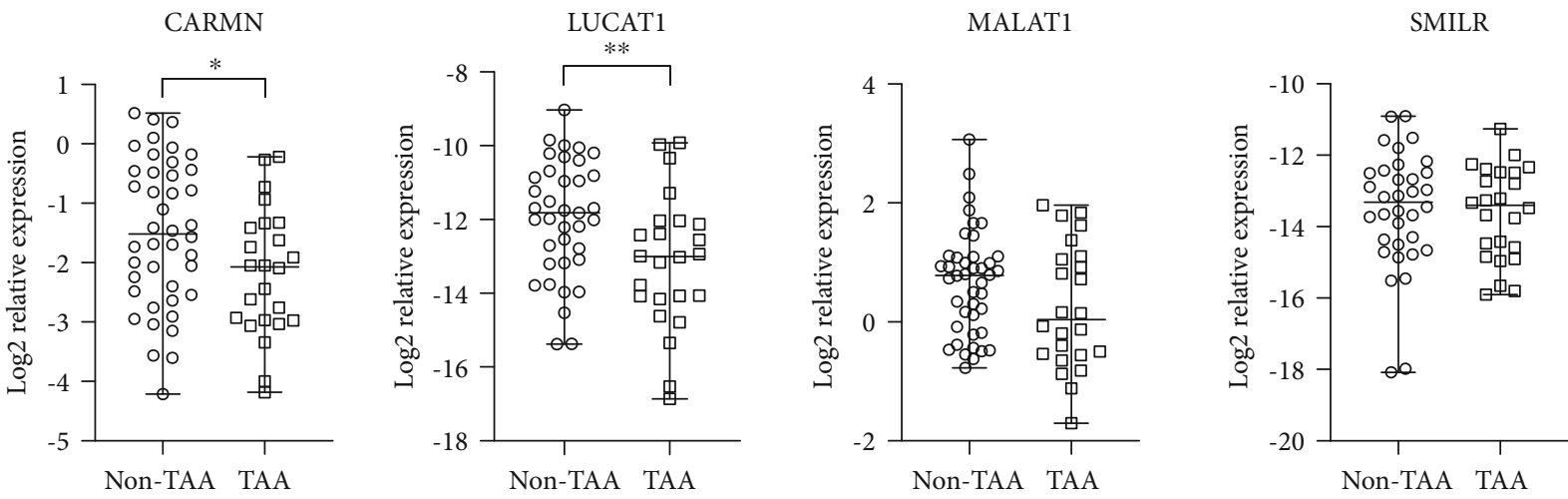

FIGURE 1: Relative expression of CARMN, LUCAT1, MALAT1, and SMILR in the human aortic wall of non-TAA and TAA patients. Whiskers indicate minimum and maximum values; horizontal lines represent median values. $*$ indicates $p<0.05 ; * *$ indicates $p<0.01$.
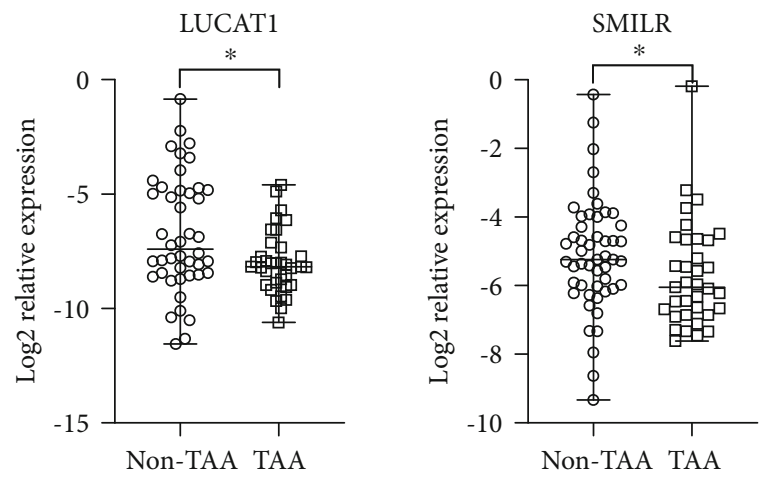

(a)
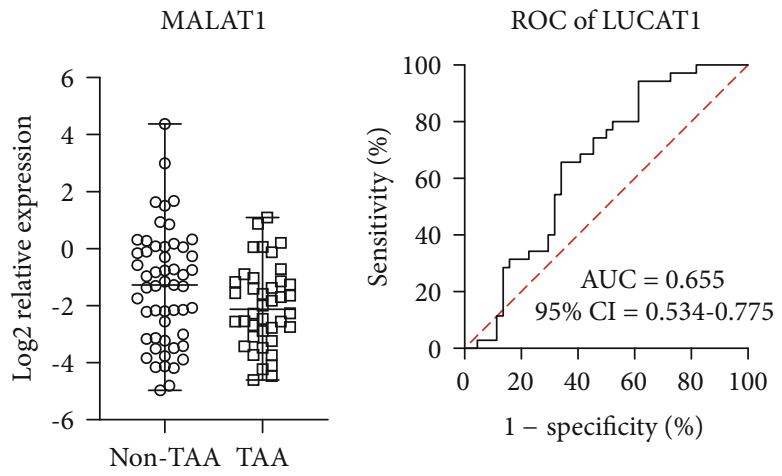

(b)

FIGURE 2: Evaluation of LUCAT1, SMILR, and MALAT1 expression in human blood plasma. (a) Relative expression of LUCAT1, SMILR, and MALAT1 in non-TAA group and TAA patients. (b) Receiver operator characteristic (ROC) curve of LUCAT1 expression in blood plasma for identification of TAA. Whiskers indicate minimum and maximum values; horizontal lines represent median values. $*$ indicates $p<0.05$. AUC: area under curve; CI: confidence interval.

associated TAA. Further studies using larger sample sizes will be needed to elucidate the relationship between LUCAT1 and BAV in TAA disease.

LUCAT1 expression in whole blood is relatively abundant compared to other human tissues; therefore, it has great potential to be used as a biomarker for the disease. Decreased expression of LUCAT1 in the blood serum of chronic heart failure patients has been associated with poor prognosis [29] and shows diagnostic potential in identifying diabetic patients with diabetic lung disease [31]. LUCAT1 is also found in blood serum exosomes of hepatocellular carcinoma patients [32]. Our study is the first one to show reduced LUCAT1 expression in the blood plasma of TAA patients and its potential to be used as a disease biomarker in the formation of the disease. A review by Trimarchi and colleagues shows that the RNA signature in a patient's blood could be used for TAA diagnosis and as a possible predictor for aortic expansion with an overall $70 \%-80 \%$ accuracy [33]. Most recent studies show the ability of circulating miRNAs to discriminate TAA patients from controls with AUC values greater than 0.8 in both blood serum [34] and plasma [35] samples.
LUCAT1 has a weaker diagnostic potential, but it could be used in combination with other clinical data for diagnosis of TAA.

We did not find significant changes in relative expression of MALAT1 and SMILR in aortic tissue samples between TAA and non-TAA patients. MALAT1 is associated with vascular inflammation as well as endothelial cell function [36] and induces aortic stiffness [37]. Increased SMILR expression is linked to VSMC proliferation in unstable atherosclerotic plaques and in plasma from patients with increased inflammation biomarkers $[8,38]$. While VSMC migration is associated with atherosclerosis and is more commonly observed in abdominal aortic aneurysms (AAA), formation of TAA is more often associated with a production of various extracellular proteases and their inhibitors as a result of increased synthetic capabilities of VSMCs. MALAT1 and SMILR could display different mechanisms of VSMC dedifferentiation, which do not play a major part in the development of TAA. Even though SMILR expression in blood plasma was significantly reduced in TAA patients compared to the non-TAA group, it did not pass the necessary threshold for the ability to identify aneurysmal disease in TAA patients. 
This study has potential limitations. Firstly, our nonTAA group for tissue analysis consists mainly of CABG patients. Since it is suggested that senescent VSMCs switch to the synthetic phenotype [39], our goal is to match TAA patients and control subjects by age. Heart donors are usually younger than TAA patients; therefore, confounding could rarely be avoided. In addition, the underlying mechanism of LUCAT1 and CARMN in the development of TAA remains unclear and requires further investigation.

\section{Conclusions}

The asymptomatic nature of TAA and lack of medicinal treatment are a driving force behind the biomarker research for the disease. We have shown that LUCAT1 and CARMN might be important players in the formation of TAA. Reduced expression of LUCAT1 shows potential to be used as a minimally invasive biomarker in blood plasma for TAA diagnosis. Further studies will be needed to elucidate the underlying mechanism of this regulatory pathway.

\section{Data Availability}

Data will be made available upon request.

\section{Conflicts of Interest}

The authors declare no conflict of interest.

\section{Authors' Contributions}

Vaiva Patamsyte was responsible for conceptualization, investigation, and writing of the original draft. Giedrius Žukovas was responsible for the methodology, data curation, and review and editing of the draft. Dovydas Gečys was responsible for the formal analysis, visualization, and review and editing of the draft. Diana Žaliaduonytè was responsible for the methodology, data curation, and review and editing of the draft. Povilas Jakuška was responsible for the investigation, data curation, and review and editing of the draft. Rimantas Benetis was responsible for conceptualization, resources, and review and editing of the draft. Vaiva Lesauskaitè was responsible for conceptualization, resources, supervision, and review and editing of the draft.

\section{Acknowledgments}

This research was funded by a grant (No. SEN-05/2016) from the Research Council of Lithuania.

\section{References}

[1] J. H. Black, C. L. Greene, and J. Y. Woo, Epidemiology, risk factors, pathogenesis and natural history of thoracic aortic aneurysm 2019November 2019, https://www.uptodate.com/ contents/epidemiology-risk-factors-pathogenesis-andnatural-history-of-thoracic-aortic-aneurysm.

[2] G. H. W. van Bogerijen, J. L. Tolenaar, V. Grassi et al., "Biomarkers in TAA-The Holy Grail," Progress in Cardiovascular Diseases, vol. 56, no. 1, pp. 109-115, 2013.
[3] S. S. Zhou, J. P. Jin, J. Q. Wang et al., "miRNAS in cardiovascular diseases: potential biomarkers, therapeutic targets and challenges," Acta Pharmacologica Sinica, vol. 39, no. 7, pp. 1073-1084, 2018.

[4] Z. Wu, M. Trenner, R. A. Boon, J. M. Spin, and L. Maegdefessel, "Long noncoding RNAs in key cellular processes involved in aortic aneurysms," Atherosclerosis, vol. 292, pp. 112-118, 2020.

[5] J. S. Mattick, "The state of long non-coding RNA biology," Non-Coding RNA, vol. 4, no. 3, p. 17, 2018.

[6] V. Simion, S. Haemmig, and M. W. Feinberg, "IncRNAs in vascular biology and disease," Vascular Pharmacology, vol. 114, pp. 145-156, 2019.

[7] D. M. Milewicz, K. M. Trybus, D. C. Guo et al., "Altered smooth muscle cell force generation as a driver of thoracic aortic aneurysms and dissections," Arteriosclerosis, Thrombosis, and Vascular Biology, vol. 37, no. 1, pp. 26-34, 2017.

[8] R. D. Bell, X. Long, M. Lin et al., "Identification and initial functional characterization of a human vascular cell-enriched long noncoding RNA," Arteriosclerosis, Thrombosis, and Vascular Biology, vol. 34, no. 6, pp. 1249-1259, 2014.

[9] M. D. Ballantyne, K. Pinel, R. Dakin et al., "Smooth muscle enriched long noncoding RNA (SMILR) regulates cell proliferation," Circulation, vol. 133, no. 21, pp. 2050-2065, 2016.

[10] A. Leung, C. Trac, W. Jin et al., "Novel long noncoding RNAs are regulated by angiotensin II in vascular smooth muscle cells," Circulation Research, vol. 113, no. 3, pp. 266-278, 2013.

[11] A. Duggirala, F. Delogu, T. G. Angelini et al., "Non coding RNAs in aortic aneurysmal disease," Frontiers in Genetics, vol. 6, 2015.

[12] Y. $\mathrm{Hu}$ and J. Hu, "Diagnostic value of circulating lncRNA ANRIL and its correlation with coronary artery disease parameters," Brazilian Journal of Medical and Biological Research, vol. 52, no. 8, p. e8309, 2019.

[13] Q. Yin, A. Wu, and M. Liu, "Plasma long non-coding RNA (lncRNA) GAS5 is a new biomarker for coronary artery disease," Medical Science Monitor, vol. 23, pp. 6042-6048, 2017.

[14] M. Azat, X. Huojiahemaiti, R. Gao, and P. Peng, "Long noncoding RNA MIAT: a potential role in the diagnosis and mediation of acute myocardial infarction," Molecular Medicine Reports, vol. 20, no. 6, pp. 5216-5222, 2019.

[15] Q. Li, P. Li, J. Su et al., "IncRNA NKILA was upregulated in diabetic cardiomyopathy with early prediction values," Experimental and Therapeutic Medicine, vol. 18, no. 2, pp. 1221$1225,2019$.

[16] H. Deng, W. Ouyang, L. Zhang, X. Xiao, Z. Huang, and W. Zhu, "lncRNA GASL1 is downregulated in chronic heart failure and regulates cardiomyocyte apoptosis," Cellular \& Molecular Biology Letters, vol. 24, no. 1, 2019.

[17] T. F. Song, L. W. Huang, Y. Yuan et al., "IncRNA MALAT1 regulates smooth muscle cell phenotype switch via activation of autophagy," Oncotarget, vol. 9, no. 4, pp. 4411-4426, 2018.

[18] Y. H. Lim, D. H. Kwon, J. Kim, W. J. Park, H. Kook, and Y. K. Kim, "Identification of long noncoding RNAs involved in muscle differentiation," PLoS One, vol. 13, no. 3, article e0193898, 2018.

[19] R. Erbel, V. Aboyans, C. Boileau et al., "2014 ESC guidelines on the diagnosis and treatment of aortic diseases: Document covering acute and chronic aortic diseases of the thoracic and abdominal aorta of the adult. The Task Force for the Diagnosis and Treatment of Aortic Diseases of the European Society of 
Cardiology (ESC)," European Heart Journal, vol. 35, no. 41, pp. 2873-2926, 2014.

[20] J. Hanley and B. McNeil, "The meaning and use of the area under a receiver operating characteristic (ROC) curve," Radiology, vol. 143, no. 1, pp. 29-36, 1982.

[21] S. Ounzain, R. Micheletti, C. Arnan et al., "CARMEN, a human super enhancer-associated long noncoding RNA controlling cardiac specification, differentiation and homeostasis," Journal of Molecular and Cellular Cardiology, vol. 89, no. Part A, pp. 98-112, 2015.

[22] Y. Li, Y. Liu, S. Liu et al., "Differential expression profile of long non-coding RNAs in human thoracic aortic aneurysm," Journal of Cellular Biochemistry, vol. 119, no. 10, pp. 7991-7997, 2018.

[23] J. E. Kontaraki, M. E. Marketou, G. E. Kochiadakis et al., "The long non-coding RNAsMHRT,FENDRRandCARMEN, their expression levels in peripheral blood mononuclear cells in patients with essential hypertension and their relation to heart hypertrophy," Clinical and Experimental Pharmacology \& Physiology, vol. 45, no. 11, pp. 1213-1217, 2018.

[24] Y. Sun, S. D. Jin, Q. Zhu et al., "Long non-coding RNA LUCAT1 is associated with poor prognosis in human nonsmall cell lung cancer and regulates cell proliferation via epigenetically repressing p21 and p57 expression," Oncotarget, vol. 8, no. 17, pp. 28297-28311, 2017.

[25] B. Luzón-Toro, R. M. Fernández, J. M. Martos-Martínez, M. Rubio-Manzanares-Dorado, G. Antiñolo, and S. Borrego, "LncRNA_LUCAT1_ as a novel prognostic biomarker for patients with papillary thyroid cancer," Scientific Reports, vol. 9, no. 1, p. 14374, 2019.

[26] Q. Zhou, Z. Hou, S. Zuo et al., "LUCAT1 promotes colorectal cancer tumorigenesis by targeting the ribosomal protein L40MDM2-p 53 pathway through binding with UBA52," Cancer Science, vol. 110, no. 4, pp. 1194-1207, 2019.

[27] Y. S. Gao, X. Z. Liu, Y. G. Zhang, X. J. Liu, and L. Z. Li, “Knockdown of long noncoding RNA LUCAT1 inhibits cell viability and invasion by regulating miR-375 in glioma," Oncology Research, vol. 26, no. 2, pp. 307-313, 2018.

[28] P. Thai, S. Statt, C. H. Chen, E. Liang, C. Campbell, and R. Wu, "Characterization of a novel long noncoding RNA, SCAL1, induced by cigarette smoke and elevated in lung cancer cell lines," American Journal of Respiratory Cell and Molecular Biology, vol. 49, no. 2, pp. 204-211, 2013.

[29] T. Li, D. Qian, J. Guoyan, and Z. Lei, “Downregulated long noncoding RNA LUCAT1 inhibited proliferation and promoted apoptosis of cardiomyocyte via miR-621/HOX13 pathway in chronic heart failure," European Review for Medical and Pharmacological Sciences, vol. 24, pp. 385-395, 2020.

[30] Q. Xia, L. Zhang, H. Yan, L. Yu, W. Shan, and H. Jiang, "LUCAT1 contributes to MYRF-dependent smooth muscle cell apoptosis and may facilitate aneurysm formation via the sequestration of miR-199a-5p," Cell Biology International, vol. 44, pp. 755-763, 2019.

[31] P. Li, N. Zhang, F. Ping, Y. Gao, and L. Cao, "IncRNA SCAL1 inhibits inducible nitric oxide synthase in lung cells under high-glucose conditions," Experimental and Therapeutic Medicine, vol. 18, pp. 1831-1836, 2019.

[32] L. Gramantieri, M. Baglioni, F. Fornari et al., "IncRNAs as novel players in hepatocellular carcinoma recurrence," Oncotarget, vol. 9, no. 80, pp. 35085-35099, 2018.
[33] S. Trimarchi, G. Sangiorgi, X. Sang et al., "In search of blood tests for thoracic aortic diseases," The Annals of Thoracic Surgery, vol. 90, no. 5, pp. 1735-1742, 2010.

[34] A. Boileau, C. L. Lino Cardenas, A. Courtois et al., "miR-5745p: a circulating marker of thoracic aortic aneurysm," International Journal of Molecular Sciences, vol. 20, no. 16, p. 3924, 2019.

[35] S. Gasiulè, V. Stankevičius, V. Patamsytè et al., "Tissue-specific miRNAs regulate the development of thoracic aortic aneurysm: the emerging role of KLF4 network," Journal of Clinical Medicine, vol. 8, no. 10, p. 1609, 2019.

[36] K. M. Michalik, X. You, Y. Manavski et al., "Long noncoding RNA MALAT1 regulates endothelial cell function and vessel growth," Circulation Research, vol. 114, no. 9, pp. 1389-1397, 2014.

[37] C. K. Yu, T. Xu, R. K. Assoian, and D. J. Rader, "Mining the stiffness-sensitive transcriptome in human vascular smooth muscle cells identifies long noncoding RNA stiffness regulators," Arteriosclerosis, Thrombosis, and Vascular Biology, vol. 38, no. 1, pp. 164-173, 2018.

[38] A. D. Mahmoud, M. D. Ballantyne, V. Miscianinov et al., "The human-specific and smooth muscle cell-enriched lncRNA SMILR promotes proliferation by regulating mitotic CENPF mRNA and drives cell-cycle progression which can be targeted to limit vascular remodeling," Circulation Research, vol. 125, no. 5, pp. 535-551, 2019.

[39] B. A. Monk and S. J. George, "The effect of ageing on vascular smooth muscle cell behaviour - a mini-review," Gerontology, vol. 61, no. 5, pp. 416-426, 2015. 